\title{
The modified fundamental equations of quantum mechanics
}

\section{in symmetric forms}

\author{
Huai-Yu Wang \\ Department of Physics, Tsinghua University, Beijing 100084, China \\ wanghuaiyu@mail.tsinghua.edu.cn
}

Keywords: Dirac equation, Klein-Gordon equation, Schrödinger equation, negative kinetic energy, Decoupled Klein-Gordon equation, negative kinetic energy Schrödinger equation.

\begin{abstract}
Up to now, Schrödinger equation, Klein-Gordon equation (KGE) and Dirac equation are believed the fundamental equations of quantum mechanics. Schrödinger equation has a defect that there is no NKE solutions. Dirac equation has positive kinetic energy (PKE) and negative kinetic energy (NKE) branches. Both branches should have low momentum, or nonrelativistic, approximations: one is Schrödinger equation and the other is NKE Schrödinger equation. KGE has two problems: it is an equation of second time derivative, and calculated density is not definitely positive. To overcome the problems, it should be revised as PKE and NKE decoupled KGEs. The fundamental equations of quantum mechanics after the modification have at least two merits. They are of unitary in that everyone contains the first time derivative and are symmetric with respect to PKE and NKE. This reflects the symmetry of the PKE and NKE matters, as well as matter and dark matter, of our universe. The problems of one-dimensional step potentials are resolved by means of the modified fundamental equations for a nonrelativistic particle.
\end{abstract}




\section{Introduction}

The fundamental equations of quantum mechanics (QM) refer to Schrödinger equation [1-4], Klein-Gordon equation (KGE) [5,6] and Dirac equation [7], which have been used up to now and are fully introduced in QM textbooks [8,9]. The latter two are relativistic quantum mechanics equations (RQMEs).

Schrödinger equation [1-4] is the first one of QM equations (QMEs) and applies to microscopic particles doing low momentum motion. The low momentum motion is usually called nonrelativistic motion. Schrödinger tried to extend his equations to the case of relativity [4], but failed, because the evaluated fine structure of hydrogen atom was not consistent with experimental one [8]. Dirac equation explained experiments almost perfectly.

In a previous work [10], we were aware of the inconsistency between Schrödinger equation and the low momentum approximations of RQMEs, and suggested the way how to remedy the inconsistency. The main result was that a relativistic particle had solutions of negative energy branch which meant that a particle could have negative kinetic energy (NKE) besides positive kinetic energy (PKE). This NKE should still be retained when the particle did nonrelativistic motion. We proposed experiments to verify the existence of the NKE.

The RQMEs actually were valid for any momentum value: the momentum could be from zero to an arbitrarily large number, i. e., they apply to both relativistic and nonrelativistic motions. Let us suppose that a particle with spin-0 does relativistic motion, so that it obeys KGE. Now, we let the particle, in some way, gradually decrease its momentum. At any momentum, it always obeys KGE, and when its momentum is very low, it still does. Meanwhile, because its momentum is very low, it also observes Schrödinger equation. Although Schrödinger equation is an approximate one, the difference between the wave functions solved from KGE and Schrödinger equation is negligible when the particle's momentum is very low. Therefore, a nonrelativistic particle with spin-0 observes both KGE and Schrödinger equation. In the same manner, we can understand that a nonrelativistic particle with spin-1/2 observes both Dirac equation and Schrödinger equation. This consideration stimulates us to notice the following problems concerning the fundamental equations of QM.

The first problem is that a particle has positive and negative energy branches if solved by Dirac equation, while it has only positive energy branch if solved by Schrödinger equation. That is to say, the negative energy branch is lost in Schrödinger equation, showing a mismatch between these two equations. The second problem is from KGE, which contains second time derivative, inconsistent with Dirac equation and Schrödinger equation.

This paper tries to solve these problems, and naturally we manage to modify the fundamental equations of QM to become correct forms. Our footstone is that Dirac equation is no doubt correct. In considering the usage and relations between these equations, we also notice two points. One is that occasionally, Dirac equation might not be used and understood correctly. The example was Klein's paradox [11-23]. This 
paradox also existed for KGE $[12,15,19,22]$. The other point is that besides KGE, there was another RQME describing the relativistic motion of spin-0, called Salpeter equation [24-41]. The form of this equation prompted us how to modify the equations of relativistic particles with spin- 0 .

This paper is arranged as follows. In section 2, the fundamental equations of QM are modified so as to get rid of the above mentioned two problems. The modified equations are uniformly of first time derivative and symmetric with respect to positive and negative energy branches. Section 3 gives an example showing that the results calculated by the modified equations differ from those by Schrödinger equation. Section 4 is our conclusions.

\section{Fundamental equations in symmetric forms}

In Newtonian mechanics of classical physics, the kinetic energy of a body is defined by

$$
K_{(+)}=\frac{p^{2}}{2 m} .
$$

Here the subscript $(+)$ refers to PKE. The momentum $\boldsymbol{p}$ is real. When a body is subject to a potential $V$, the body's energy is

$$
E_{(+)}=\frac{p^{2}}{2 m}+V>V
$$

In special relativity, a body's energy is expressed by

$$
E_{(+)}=\sqrt{m^{2} c^{4}+c^{2} \boldsymbol{p}^{2}} .
$$

When the body is subject to a potential $V$, its energy becomes

$$
E_{(+)}=\sqrt{m^{2} c^{4}+c^{2} \boldsymbol{p}^{2}}+V>V .
$$

When the momentum is very low, Eqs. (2.3) and (2.4) can be respectively approximated to (2.1) and (2.2) plus a static energy $m c^{2}$. Please note that in both Eqs. (2.2) and (2.4), it is impossible for a particle's energy to be $E_{(+)}<V$, which is a remarkable feature of classical mechanics.

Here it should be noted that Eqs. (2.1) and (2.2) are valid only for nonrelativistic motion. While Eqs. (2.3) and (2.4) stand for any momentum value, i. e., the momentum can be from zero to an arbitrary large number. When the momentum is very low, the particle's energy can be expressed by either of Eqs. (2.2) and (2.4) because, besides a constant static energy, they merely differ by a negligible small quantity. Using (2.1) and (2.2) is simpler, but using (2.3) and (2.4) is more precise.

In QM, the motion of a particle when its momentum was very low was believed to obey Schrödinger equation. 


$$
\mathrm{i} \hbar \frac{\partial}{\partial t} \psi=\left(-\frac{\hbar^{2}}{2 m} \nabla^{2}+V\right) \psi
$$

Then, KGE was proposed to describe the relativistic motion of spin-0 particles.

$$
\left(\mathrm{i} \hbar \frac{\partial}{\partial t}-V\right)^{2} \Psi=\left(m^{2} c^{4}-c^{2} \hbar^{2} \nabla^{2}\right) \Psi .
$$

Shortly afterwards, Dirac equation was developed, which was for the relativistic motion of spin-1/2 particles.

$$
\mathrm{i} \hbar \frac{\partial}{\partial t} \Psi=\left(-\mathrm{i} c \hbar \alpha \cdot \nabla+m c^{2} \beta+V\right) \Psi .
$$

Equations (2.5), (2.6) and (2.7) are fundamental equations of QM being used nowadays, and are listed in Table I. In Table 1, some problems related to Schrödinger equation and KGE are briefly mentioned. Here we have a detailed analysis of these problems.

We first inspect Dirac equation. From it the energy of a free relativistic particle is expressed by

$$
E_{( \pm)}= \pm \sqrt{m^{2} c^{4}+c^{2} \boldsymbol{p}^{2}}
$$

\begin{tabular}{|c|c|c|c|}
\hline \multirow{2}{*}{$\begin{array}{l}\text { Relati- } \\
\text { vistic } \\
\text { motion }\end{array}$} & $\begin{array}{l}\text { Spin-1/2 } \\
\text { particles }\end{array}$ & $\begin{array}{l}\text { Dirac equation } \\
\mathrm{i} \hbar \frac{\partial}{\partial t} \Psi=\left(c \boldsymbol{\alpha} \cdot \boldsymbol{p}+m c^{2} \beta+V\right) \Psi\end{array}$ & \\
\hline & $\begin{array}{l}\text { Spin-0 } \\
\text { particle }\end{array}$ & $\begin{array}{l}\text { Klein-Gordon equation } \\
\left(\mathrm{i} \hbar \frac{\partial}{\partial t}-V\right)^{2} \psi=\left(m^{2} c^{4}+c^{2} \boldsymbol{p}^{2}\right) \psi\end{array}$ & $\begin{array}{l}\text { Problems: second time } \\
\text { derivative, probability } \\
\text { density being not } \\
\text { definitely positive. }\end{array}$ \\
\hline \multicolumn{2}{|c|}{$\begin{array}{l}\text { Nonrelativistic } \\
\text { motion }\end{array}$} & $\begin{array}{l}\text { Schrödinger equation } \\
\mathrm{i} \hbar \frac{\partial}{\partial t} \psi=\left(\frac{1}{2 m} \boldsymbol{p}^{2}+V\right) \psi\end{array}$ & $\begin{array}{l}\text { Problems: lack of } \\
\text { negative energy } \\
\text { branch, there is no } \\
\text { solution when } V \rightarrow-V .\end{array}$ \\
\hline
\end{tabular}

There are two energy branches. The positive branch $E_{(+)}$is exactly the same as (2.3), i. e., it has classical correspondence.

Table 1. Fundamental equations of QM being used, where $\boldsymbol{p}=-\mathrm{i} \hbar \nabla$.

The negative branch does not have classical correspondence. Its explicit form is

$$
E_{(-)}=-\sqrt{m^{2} c^{4}+c^{2} \boldsymbol{p}^{2}} \text {. }
$$

It is just the contrary number of (2.3). Thus, since (2.3) contains positive static energy and PKE, naturally, (2.9) contains negative static energy and NKE. When the particle is subject to a potential $V$, then the positive branch becomes Eq. (2.4) and the negative 
branch becomes

$$
E_{(-)}=-\sqrt{m^{2} c^{4}+c^{2} \boldsymbol{p}^{2}}+V<V .
$$

When the momentum is very low, Eq. (2.10) can be approximated, with the static energy dropped, to be

$$
E_{(-)}=-\frac{p^{2}}{2 m}+V<V
$$

We denote

$$
K_{(-)}=-\frac{p^{2}}{2 m} .
$$

Here the NKE of low momentum is in the form of (2.12) which is rigorously derived from (2.9), and the momentum $\boldsymbol{p}$ is real. Please note that in both Eqs. (2.10) and (2.11), the energy $E_{(-)}$is always less than potential $V$. This feature comes from QM. Conversely, if a particle's energy is larger than its potential, the energy should be expressed by Eqs. (2.2) or (2.4), and if a particle's energy is less than its potential, the energy should be expressed by Eqs. (2.10) or (2.11).

When one takes transformation

$$
\Psi=\psi_{(+)} \mathrm{e}^{-\mathrm{i} m c^{2} t / \hbar}
$$

in Dirac equation, then the wave function $\psi_{(+)}$satisfies Schrödinger equation (2.5) in low momentum approximation. That is to say, Schrödinger equation (2.5) is a low momentum approximation of Dirac equation.

Now let us consider such a situation: a free particle is moving with very low momentum. On one hand, its motion obeys Schrödinger equation from which the particle's energy solved is Eq. (2.2). On the other hand, Dirac equation is still applicable, from which the particle's energy has two branches as shown by Eq. (2.8). So, in Schrödinger equation, the negative energy branch is absent.

The author believes that Dirac equation is correct, and the negative energy branch should be treated on an equal footing as the positive one. Thereby, the negative branch should be retained even in nonrelativistic motion. Fortunately, this can be achieved by the transformation

$$
\Psi=\psi_{(-)} \mathrm{e}^{\mathrm{i} m c^{2} t / \hbar}
$$

By this transformation and taking low momentum approximation, we obtain

$$
\mathrm{i} \hbar \frac{\partial}{\partial t} \psi_{(-)}=\left(\frac{\hbar^{2}}{2 m} \nabla^{2}+V\right) \psi_{(-)} .
$$

From this equation, a free particle has energy (2.12) which is of NKE. Therefore, Eq. (2.15) is called NKE Schrödinger equation. Hence, Eq. (2.15), as another low momentum approximation of Dirac equation, embodies the negative energy branch and 
applies to the cases of $E_{(-)}<V$, while Schrödinger equation applies to the cases of $E_{(+)}>V$. The combination of the two equations inherit the properties of Dirac equation in nonrelativistic motion and are listed in the bottom row in Table 2.

Here, we mention that Schrödinger himself already put down NKE Schrödinger equation. The primary time-dependent equations he wrote were in the following form [4].

$$
\frac{\hbar^{2}}{2} \nabla^{2} \psi-V \psi \mp \mathrm{i} \hbar \frac{\partial \psi}{\partial t}=0
$$

That is to say, he gave Schrödinger equation and NKE Schrödinger equation simultaneously at the very beginning. Of cause, at that time, he was unable to recognize the difference in physical meanings between of difference in sign in Eq. (2.16), and neither were others. Schrödinger just firmly believed that both signs in (2.16) were right in mathematics. Later, people adopted, as Schrödinger himself did, the form corresponding to PKE.

We stress that Schrödinger equation is merely applicable to nonrelativistic motion, while Dirac equation is valid for all momenta so that it is a comprehensive one. It is hard to find the flaws of Schrödinger equation by inspecting itself. When starting from Dirac equation and taking its low momentum approximation we can find the problems of Schrödinger equation.

Table 2. Modified fundamental equations of QM, where $H_{0}=\sqrt{m^{2} c^{4}-c^{2} \hbar^{2} \nabla^{2}}$.

\begin{tabular}{|l|l|c|c|}
\hline & & In PKE region, $E>V$ & In NKE region, $E<V$ \\
\hline \multirow{2}{*}{$\begin{array}{l}\text { Relati- } \\
\text { vistic } \\
\text { motion }\end{array}$} & $\begin{array}{l}\text { Spin-1/2 } \\
\text { particle }\end{array}$ & \multicolumn{2}{|c|}{$\mathrm{i} \hbar \frac{\partial}{\partial t} \Psi=\left(-\mathrm{i} c \hbar \boldsymbol{\alpha} \cdot \nabla+m c^{2} \beta+V\right) \Psi$} \\
\cline { 2 - 4 } & $\begin{array}{l}\text { Spin-0 } \\
\text { particle }\end{array}$ & $\begin{array}{l}\text { PKE decoupled KGE } \\
\text { (Salpeter equation }) \\
\mathrm{i} \hbar \frac{\partial}{\partial t} \psi_{(+)}=\left(H_{0}+V\right) \psi_{(+)}\end{array}$ & $\mathrm{i} \hbar \frac{\partial}{\partial t} \psi_{(-)}=\left(-H_{0}+V\right) \psi_{(-)}$ \\
\hline \multirow{2}{*}{$\begin{array}{l}\text { Nonrelativistic } \\
\text { motion }\end{array}$} & $\mathrm{Schrödinger} \mathrm{equation}$ & $\mathrm{NKE} \mathrm{Schrödinger} \mathrm{equation}$ \\
& $\mathrm{i} \hbar \frac{\partial}{\partial t} \psi_{(+)}=\left(-\frac{\hbar^{2}}{2 m} \nabla^{2}+V\right) \psi_{(+)}$ & $\mathrm{i} \hbar \frac{\partial}{\partial t} \psi_{(-)}=\left(\frac{\hbar^{2}}{2 m} \nabla^{2}+V\right) \psi_{(-)}$ \\
\hline
\end{tabular}

Now we turn to KGE (2.6). It has a severe problem coming from the fact that Eq. (2.6) contains the second derivatives with respect to time. Let us consider a spin-0 particle doing very low momentum motion. It should obey Schrödinger equation which contains the first time derivative. Suppose that we are able to accelerate the particle in 
some way until it does relativistic motion. Then it should obey KGE. We would like to ask a question: in the course of the increase of the particle's momentum, at which momentum the equation that the particle satisfies transits from the first time derivative one to the second time derivative one, and why? We are unable to give a satisfactory answer.

Because KGE contains second time derivative, in order to solve the wave function, the initial conditions needed are the initial value of the wave function

$$
\psi(\boldsymbol{r}, t=0)
$$

and the initial value of the first time derivative of the wave function

$$
\left[\frac{\partial}{\partial t} \psi(\boldsymbol{r}, t)\right]_{t=0} .
$$

By contrast, in solving Schrödinger equation, only condition (2.17) is needed.

A contradiction arises when a spin- 0 particle does nonrelativistic motion. On one hand, its wave functions can be solved by Schrödinger equation under the initial condition (2.17). On the other hand, it should also be solved from KGE, but with an additional initial condition (2.18). Whether is the condition (2.18) necessary or not on earth?

A consequence of the second time derivative of KGE was that the probability density derived from this equation could be negative, the famous negative probability difficulty $[8,9]$.

This problem demonstrates that one of Eqs. (2.5) and (2.6) is incorrect. It is believed that Schrödinger equation correctly describes the motion of particles for low momenta and energies $E>V$, and it is a low momentum approximation of Dirac equation.

Thus, it is concluded that KGE is not the proper one for relativistic particles with spin- 0 . Let us determine the proper ones.

In the author's previous papers [10,42], we have pointed out that when the potential was piecewise constant, KGE could be factorized as

$$
\left(\mathrm{i} \hbar \frac{\partial}{\partial t}+H_{0}+V\right)\left(\mathrm{i} \hbar \frac{\partial}{\partial t}-H_{0}+V\right) \psi=0,
$$

where

$$
H_{0}=\sqrt{m^{2} c^{4}-c^{2} \hbar^{2} \nabla^{2}} .
$$

The order of the two parentheses in Eq. (2.19) can be exchanged. In this way, we obtained two decoupled equations.

$$
\mathrm{i} \hbar \frac{\partial}{\partial t} \psi_{(+)}=\left(\sqrt{m^{2} c^{4}-c^{2} \hbar^{2} \nabla^{2}}+V\right) \psi_{(+)}
$$

and

$$
\mathrm{i} \hbar \frac{\partial}{\partial t} \psi_{(-)}=\left(-\sqrt{m^{2} c^{4}-c^{2} \hbar^{2} \nabla^{2}}+V\right) \psi_{(-)} .
$$


Obviously, the energy of a particle satisfying Eq. (2.21) is related to Eq. (2.4), and that satisfying (2.22) related to (2.10). Therefore, Eq. (2.21) is called PKE decoupled KGE and (2.22) called NKE decoupled KGE. Using these forms, we solved Klein's paradox [42] for spin-0 particles.

Equations (2.21) and (2.22) are achieved when the potential is piecewise constant. We extend them by allowing the potential to be arbitrary, and think that they are the correct equations that relativistic particles with spin-0 should obey. From now on, the potential in Eqs. (2.21) and (2.22) can be arbitrary. Equation (2.21) was in fact suggested before [24-26] and called Salpeter equation. There have been investigations of Salpeter equation with various potentials [27-41]. Yndurain proved that Eq. (2.21) was of relativistic invariance [24], as a relativistic equation should be.

Equations (2.21) and (2.22) are of the following merits. First, their low momentum approximations are naturally Schrödinger equation and NKE Schrödinger equation, respectively. Explicitly, by use of Eq. (2.13) in (2.21) and by use of (2.14) in (2.22), we can obtain Eqs. (2.5) and (2.15) after taking low momentum approximations. These processes are the same as those of deriving Eqs. (2.5) and (2.15) from Dirac equation. Alternatively, one can also expand the square root of $H_{0}$ (2.20), makes low momentum approximation and drops the constant terms $\pm m c^{2}$, so as to obtain Eqs. (2.5) and (2.15). Second, the forms of Eqs. (2.21) and (2.22) lead to correct expressions of probability avoiding the difficulty of negative probability [10]. Third, these two equations helped us to correctly calculate the reflection coefficient of a relativistic particle with spin-0 through one-dimensional step potential [42]. The achieved reflection curves were qualitatively the same as those of a Dirac particle. Besides, it is believed that the decoupled KGEs will have more usage. For instance, the Wigner function of Salpeter equation was explored [43].

Equations (2.21) and (2.22) are listed in Table 2 which thus gathers all the modified fundamental QMEs. The equations listed in Table 2 have uniformly the first time derivative so that everyone can be written in the form of

$$
\mathrm{i} \hbar \frac{\partial}{\partial t} \psi=H \psi
$$

Microscopic particles with PKE can, by means of interactions between them, compose macroscopic PKE bodies which observe classical mechanics. In [45], the author obtained Newtonian mechanics and relativistic mechanics from Schrödinger equation and PKE decoupled KGE under macroscopic approximation.

Naturally, microscopic NKE particles can also, by means of interactions between them, compose macroscopic bodies. In [45], the author obtained the equations of motion for NKE bodies from NKE Schrodinger equation and PKE decoupled NGE under macroscopic approximation. They are called respectively Newtonian mechanics and relativistic mechanics for NKE bodies, as listed in Table 3 here. In that work, the macroscopic approximation was taken. After that, I was aware that the forms of decoupled KGEs (21) and (22) above can be stand for microscopic particles. 
Our universe is of good symmetry with respect to PKE and NKE matters. Tables 2 and 3 show that symmetry of fundamental equations of motion. The author's opinion is that Schrödinger equation, PKE decoupled KGE and the PKE solutions of Dirac equation describe the motion of PKE matter we have studied so far. While NKE Schrödinger equation, NKE decoupled KGE and the NKE solutions of Dirac equation describe the motion of dark matter which is of NKE. Personally, NKE matter is a synonym of dark matter. In a previous work [44], the fundamental formalism of the statistical mechanics and thermodynamics for NKE systems was given. In the field of statistical mechanics and thermodynamics, there is also good symmetry with respect to PKE and NKE systems.

In next section, we are going to give an example to show the discrepancy between the results obtained by nonrelativistic equations in Tables 1 and 2 .

Table 3. Laws of motion for PKE and NKE matters. RM refers to relativistic motion.

\begin{tabular}{|c|c|c|c|c|c|}
\hline Matter & $\begin{array}{l}\text { Laws of } \\
\text { motion }\end{array}$ & & & PKE matter & NKE matter \\
\hline \multirow{2}{*}{$\begin{array}{l}\text { Macro- } \\
\text { scopic } \\
\text { body }\end{array}$} & \multirow{2}{*}{$\begin{array}{l}\text { Classical } \\
\text { mechanics }\end{array}$} & \multicolumn{2}{|c|}{$\begin{array}{l}\text { Nonrelativistic } \\
\text { motion }\end{array}$} & $\begin{array}{l}\text { Newtonian } \\
\text { mechanics for } \\
\text { PKE bodies }\end{array}$ & $\begin{array}{l}\text { Newtonian } \\
\text { mechanics for } \\
\text { NKE bodies }\end{array}$ \\
\hline & & \multicolumn{2}{|l|}{$\mathrm{RM}$} & $\begin{array}{l}\text { Relativistic } \\
\text { mechanics for } \\
\text { PKE bodies }\end{array}$ & $\begin{array}{l}\text { Relativistic } \\
\text { mechanics for } \\
\text { NKE bodies }\end{array}$ \\
\hline \multirow{3}{*}{$\begin{array}{l}\text { Micro- } \\
\text { scopic } \\
\text { particle }\end{array}$} & \multirow{3}{*}{$\begin{array}{l}\text { Quantum } \\
\text { mechanics }\end{array}$} & \multicolumn{2}{|c|}{$\begin{array}{l}\text { Nonrelativistic } \\
\text { motion }\end{array}$} & $\begin{array}{l}\text { Schrödinger } \\
\text { equation }\end{array}$ & $\begin{array}{l}\text { NKE } \\
\text { Schrödinger } \\
\text { equation }\end{array}$ \\
\hline & & $\mathrm{RM}$ & $\begin{array}{l}\text { Spin-0 } \\
\text { particles }\end{array}$ & $\begin{array}{l}\text { Salpeter } \\
\text { equation }\end{array}$ & $\begin{array}{l}\text { NKE } \\
\text { decoupled } \\
\text { KGE }\end{array}$ \\
\hline & & & $\begin{array}{l}\text { Spin-1/2 } \\
\text { particles }\end{array}$ & \multicolumn{2}{|c|}{ Dirac equation } \\
\hline
\end{tabular}

\section{Re-calculation of reflection coefficient of a particle by a one-dimensional step potential}

\subsection{An infinitely wide potential}

The potential is

$$
V(x)=\left\{\begin{array}{c}
0, x \leq 0 \\
V_{0}, x>0
\end{array} .\right.
$$

Please see Fig. 1(a). A particle with energy $E$ and momentum of $q$ is incident from the left and moves rightwards. Let us calculate the reflection coefficient. 

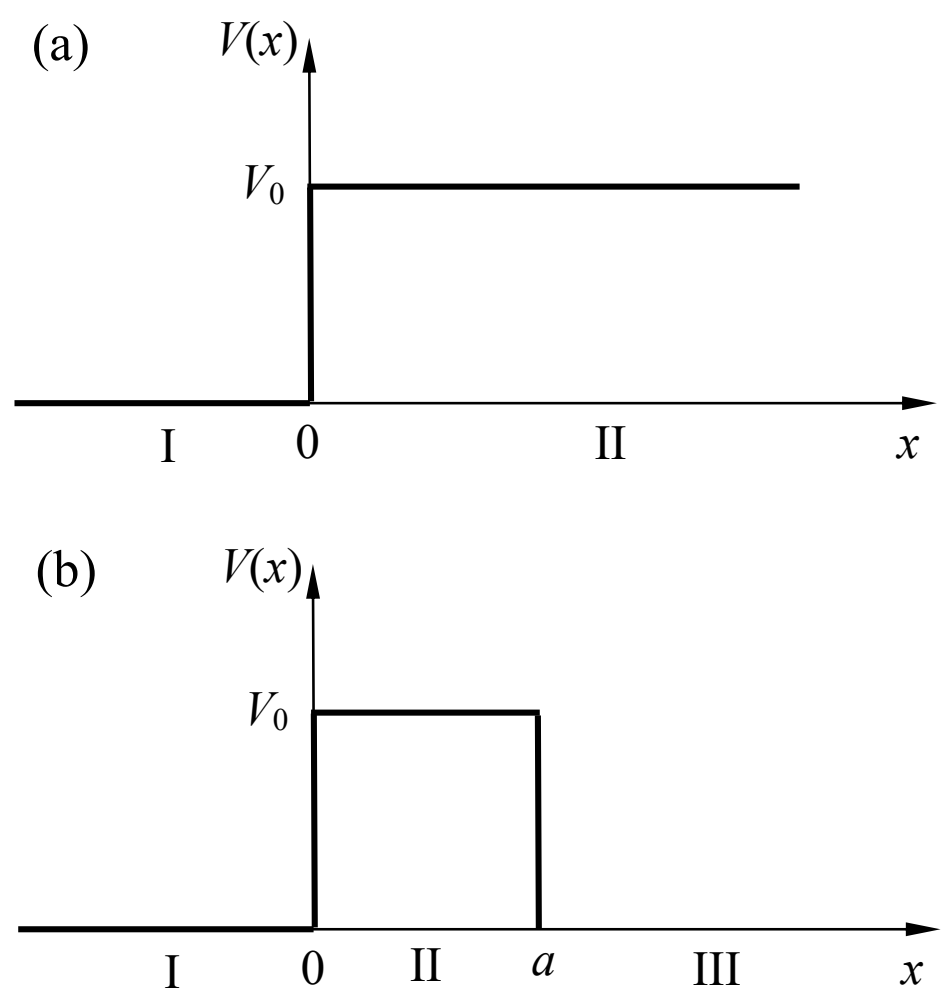

Fig. 1. One-dimensional potential barrier with height $V_{0}$. (a) Infinitely wide. (b) Finitely wide with width $a$.

At first, we retrospect what was done in usual QM textbooks. Actually, Schrödinger equation in Table 1 was used.

In region I, $x<0$, the particle's energy and its wave function are respectively

$$
E=q^{2} / 2 m
$$

and

$$
\psi_{\mathrm{I}}=\mathrm{e}^{\mathrm{i} q x / \hbar}+B \mathrm{e}^{-\mathrm{i} q x / \hbar} .
$$

The two terms in (3.3) are respectively incident and reflective waves, with $B$ being reflection amplitude. In region II, $x>0$, its energy and wave function are believed to be

$$
E=p^{2} / 2 m+V_{0}
$$

and

$$
\psi_{\text {II }}=F \mathrm{e}^{\mathrm{i} p x / \hbar},
$$

with $F$ being transmission amplitude. At the boundary $x=0$, the wave function should be continuous and smooth. These conditions lead to

$$
1+B=F
$$

and

$$
q(1-B)=p F
$$


From Eqs. (3.6) the reflection amplitude is obtained:

$$
B=\frac{q-p}{q+p}
$$

When $E>V_{0}, p$ is real and reflection coefficient is

$$
R=|B|^{2}=\left(\frac{V_{0}}{2 E-V_{0}+2 \sqrt{E\left(E-V_{0}\right)}}\right)^{2} .
$$

Numerical results are depicted by the dashed line in Fig. 2(a).

As $E<V_{0}$, it seemed from (3.4) that

$$
p=\mathrm{i} \mu
$$

was an imaginary number, and hence the reflection coefficient calculated was

$$
R=1 \text {, }
$$

i. e., the particle totally reflected. This was because in the region $x>0$ the wave function was thought decaying exponentially. This conclusion seemed plausible, but was not correct.

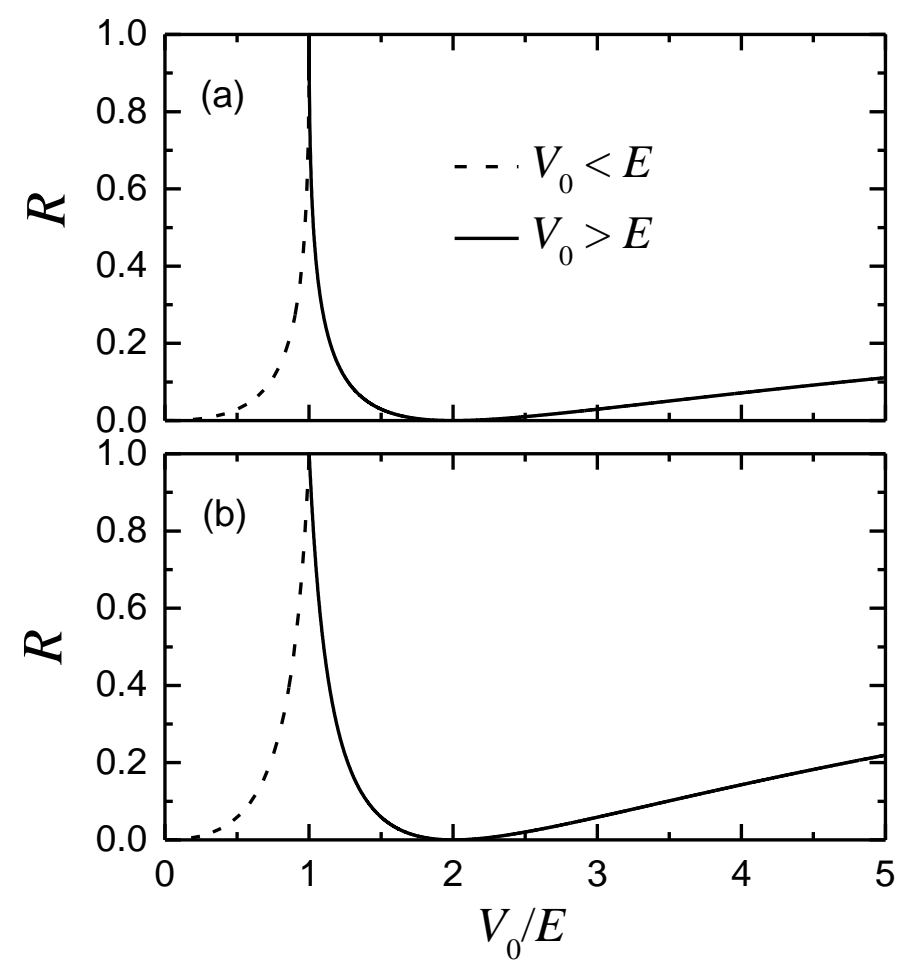

Fig. 2. Reflection coefficient $R=|B|^{2}$. (a) The potential barrier is Fig. 1(a). Dashed line is calculated by Eq. (3.8) and solid line Eq. (3.14). (b) The potential barrier is Fig. 1(b), and $\cot (p a / \hbar)=1 / 2$ is taken. Dashed line is calculated by Eq. (3.26) and solid 
line Eq. (3.32).

As a matter of fact, Table 2 tells us that in the case of $E<V_{0}$, NKE Schrödinger equation (2.15) should be employed, so that Eq. (3.4) should be replaced by (2.11).

Hence in region II, $x>0$, the equation should be

$$
\frac{\hbar^{2}}{2 m} \frac{\mathrm{d}^{2}}{\mathrm{~d} x^{2}} \psi_{\mathrm{II}}+V_{0} \psi_{\mathrm{II}}=E \psi_{\mathrm{II}} .
$$

The wave function is

$$
\psi_{\mathrm{II}}=F \mathrm{e}^{\mathrm{i} p x / \hbar} .
$$

It is a plane wave with the same form as (3.5). Correspondingly, the energy-momentum relation of the particle is

$$
E=-p^{2} / 2 m+V_{0} .
$$

Thus, $p$ is real and Eq. (3.9) is incorrect. Please compare Eqs. (3.4) and (3.13). They are manifestation of Eqs. (2.2) and (2.11). In Eq. (3.13), $E-V_{0}=-p^{2} / 2 m$ is the kinetic energy (NKE). Energy is always kinetic energy plus potential energy, no matter whether the kinetic energy is positive or negative. Please also notice that the discrepancy of (3.5) and (3.12) is that in the latter the momentum is real.

In region $x<0$, Eqs. (3.2) and (3.3) remain valid.

We have now simultaneous equations (3.3) and (3.12) plus energy-momentum relations (3.2) and (3.13). The boundary conditions have exactly the same forms of (3.6). The resultant reflection coefficient is

$$
R=|B|^{2}=\left(\frac{V_{0}-2 E}{V_{0}+2 \sqrt{E\left(V_{0}-E\right)}}\right)^{2} .
$$

Numerical results are plotted in Fig. 2(a) by solid line. The discussion of the curves in Fig. 2(a) is in the next subsection.

\subsection{A finitely wide potential}

The potential is of the form of Fig. 1(b).

$$
V(x)=\left\{\begin{array}{cc}
0, & x \leq 0 \\
V_{0}, & 0<x \leq a . \\
0, & x>a
\end{array}\right.
$$

A particle with energy $E$ and momentum $q$ is incident from $-\infty$ and moves rightwards.

We first recall what has been down in QM textbooks. forms.

In regions I and III, the wave functions are respectively written in the following 


$$
\begin{aligned}
& \psi_{\mathrm{I}}=\mathrm{e}^{\mathrm{i} q x / \hbar}+B \mathrm{e}^{-\mathrm{i} q x / \hbar}, x<0 . \\
& \psi_{\text {III }}=G \mathrm{e}^{\mathrm{i} q x / \hbar}, x>a .
\end{aligned}
$$

The energy-momentum relation is

$$
E=q^{2} / 2 m \text {. }
$$

In region II, one has to distinguish the cases of $V_{0}<E$ and $V_{0}>E$.

1. $V_{0}<E$

In region II, the wave function can be written as

$$
\psi_{\text {II }}=F_{1} \mathrm{e}^{\mathrm{i} p x / \hbar}+F_{2} \mathrm{e}^{-\mathrm{i} p x / \hbar}, 0<x<a .
$$

The energy-momentum relation is

$$
E=p^{2} / 2 m+V_{0} .
$$

The simultaneous three functions (3.16), (3.17) and (3.19) should meet the conditions that at boundaries $x=0$ and $x=a$ the wave function and its derivative are continuous. Then, the following four equations are obtained.

$$
\begin{aligned}
& 1+B=F_{1}+F_{2} . \\
& F_{1} \mathrm{e}^{\mathrm{i} p a / \hbar}+F_{2} \mathrm{e}^{-\mathrm{i} p a / \hbar}=G \mathrm{e}^{\mathrm{i} q a / \hbar} . \\
& q(1-B)=p\left(F_{1}-F_{2}\right) . \\
& p\left(F_{1} \mathrm{e}^{\mathrm{i} p a / \hbar}-F_{2} \mathrm{e}^{-\mathrm{i} p a / \hbar}\right)=q G \mathrm{e}^{\mathrm{i} q a / \hbar} .
\end{aligned}
$$

From these four equations and with the help of Eqs. (3.18) and (3.20), the reflection amplitude is solved.

$$
B=\frac{V_{0}}{2 E-V_{0}+2 \mathrm{i} \sqrt{E\left(E-V_{0}\right)} \cot (p a / \hbar)} .
$$

The reflection coefficient $R=|B|^{2}$ is

$$
R=\frac{V_{0}^{2}}{\left(2 E-V_{0}\right)^{2}+4 E\left(E-V_{0}\right) \cot ^{2}(p a / \hbar)} .
$$

Numerical results are potted by dashed line in Fig. 2(b).

From Eq. (3.26), it is seen that as the barrier height is fixed, $R$ oscillates with the barrier width $a$.

$$
\text { As } p a / \hbar=n \pi, R=0 \text {. }
$$

This is resonant transmission. In this case, from Eqs. (3.21)-(3.24) the coefficients are 
solved:

$$
B=0, G=1, F_{1}=\frac{1}{2}\left(1+\frac{q}{p}\right), F_{2}=\frac{1}{2}\left(1-\frac{q}{p}\right) .
$$

The reflection maxima are as follows.

$$
\text { As } p a / \hbar=\left(n+\frac{1}{2}\right) \pi, \quad R=\frac{V_{0}^{2}}{\left(2 E-V_{0}\right)^{2}} \text {. }
$$

2. $V_{0}>E$

In this case, according to QM textbooks, the energy-momentum relation is still Eq. (3.20), from which the momentum has to be an imaginary number, $p=\mathrm{i} k$. By the boundary conditions at $x=0$ and $x=a$, the reflection coefficient is solved to be

$$
R=\frac{V_{0}^{2}}{\left(2 E-V_{0}\right)^{2}+4 E\left(V_{0}-E\right) \operatorname{coth}^{2}(k a / \hbar)} .
$$

As $a \rightarrow \infty, R \rightarrow 1$. This is the conclusion in QM textbooks.

However, in the viewpoint of present work, in region II, the energy-momentum relation should be

$$
E=-p^{2} / 2 m+V_{0}
$$

and the wave function is still of the form of Eq. (3.20). Therefore, in this case, we have wave functions Eqs. (3.16), (3.17) and (3.19) available, but the energy-momentum relationships should be Eqs. (3.18) and (3.30). By the boundary conditions at $x=0$ and $x=a$, the reflection coefficient is solved to be

$$
R=\frac{\left(2 E-V_{0}\right)^{2}}{V_{0}^{2}+4 E\left(V_{0}-E\right) \cot ^{2}(p a / \hbar)} .
$$

Numerical results are plotted by solid line in Fig. 2(b). The discussion of curves on Fig. 2 (b) is in the next subsection.

Under fixed barrier height, the reflection coefficient oscillates with the barrier width, which is the same behavior as (3.26). The condition of resonant transmission is also (3.27) and under the conditions the coefficients are the same as (3.28)

$$
\text { As } p a / \hbar=\left(n+\frac{1}{2}\right) \pi, R=\left(1-\frac{2 E}{V_{0}}\right)^{2} \text {. }
$$

This is the reflection maximum.

\subsection{Discussions}

It is seen from the two panels of Fig. 2 that the reflection coefficient curves are qualitatively the same, and they have following common features.

As $V_{0}=0$, there is no barrier so that $R=0$. As $V_{0}$ increases starting from zero to 
$E$, reflection coefficient $R$ rises from 0 to 1 . At $V_{0}=E, p=0$. That is to say, the particle cannot move in the barrier, so that it reflects totally.

In the range $0<V_{0}<2 E$, there is a symmetry: the exchange $V_{0}-E \Leftrightarrow E-V_{0}$ makes Eq. (3.14) become (3.8) and vice versa, and makes Eq. (3.26) become (3.31) and vice versa. This is reflected in Fig. 2 that within $0<V_{0} / E<2$, the curves are symmetric with respect to $V_{0} / E=1$. As $V_{0}=2 E$, it is easily derived from (3.2) and (3.13), as well as from (3.18) and (3.20), that $p=q$. That is to say, the value of the momentum of the particle does not alter when it goes through the potential wall, as if there is no force acting on it. This situation is equivalent to that it is not scattered, and is similar to the case of "complete impedance matching" in electromagnetic materials. Therefore, the transmission coefficient is 1 .

As $V_{0}>2 E, R$ again rises with $V_{0}$ monotonically. As $V_{0} \rightarrow \infty, R \rightarrow 1$. Facing the infinitely high barrier, the particle has to reflect totally.

In a previous paper [42], we have solved the same potential problems for relativistic particles by means of the equations listed in Table 2 here. Now we compare the curves there with those in Figs. 2 here. The qualitative behaviors are the same. There is always a transmission alley at $V_{0}=2 E$. For a finitely wide potential barrier, the resonant transmission conditions are also (3.27).

In all cases as $V_{0}>2 E, R$ rises from zero monotonically. For a Dirac particle, when $V_{0} \rightarrow \infty$, roughly speaking, $R \rightarrow m^{2} c^{4} / E^{2}<1$. That is to say, even facing an infinitely high potential, there is still some probability for the particle to transmit, and the greater the particle's energy or the less its static mass, the greater the transmission probability, which might provide an insight why neutrinos could transmit through almost everywhere [42].

The discrepancies between relativistic and nonrelativistic particles when $E<V$ are that the energy of the former has a gap while that of the latter have not.

For a relativistic particle, when the energy-momentum relationship meets $c^{2} p^{2}=(E-V)^{2}+m^{2} c^{4}<0$, there is an energy gap in the range $\left[-m c^{2}+V, m c^{2}+V\right]$ with a width $2 m c^{2}$. Within this gap, the momentum $p$ is an imaginary number. Suppose that the height of the step potential barrier is within $-m c^{2}+E<V<m c^{2}+E$. The wave functions in the barrier are of exponential forms. For an infinitely wide potential, the reflection coefficient curves within this range is 1 , called reflection platform; for a 
finitely wide potential, the curve in this range is less than 1 [42].

For a nonrelativistic particle, the energy has no gap, see Eqs. (3.4), (3.13), (3.20) and (3.30). There is no possibility for the momentum $p$ to be imaginary. The curves within $-m c^{2}+E<V_{0}<m c^{2}+E$ for a relativistic particle contract to a point at $V_{0}=E$ in Figs. 2(a) and 2(b).

The usual treatment of tunneling problem in QM textbooks gives (3.29) that is not correct, since NKE Schrödinger equation is not touched. Nevertheless, no one has noticed that because no superficial contradiction appeared. As for relativistic motion, neglecting the negative energy solution caused the reflection coefficient larger than 1 , so that Klein's paradox emerged.

Here, we emphasize that in the regions $E<V$ the equation, i. e., Schrödinger equation, is not generated out of nothing but derived from Dirac equation rigorously by means of transformation (2.14).

\section{Conclusions}

The unmodified fundamental equations of quantum mechanics (QM) are Dirac equation, Klein-Gordon equation (KGE) and Schrödinger equation. The fundamental equations are modified as follows. Dirac equation for spin-1/2 particles remains unchanged, which has positive kinetic energy (PKE) and negative kinetic energy (NKE) branches. A relativistic particle with spin-0 observes PKE (NKE) decoupled KGE for $E>V(E<V)$. A nonrelativistic particle observes Schrödinger equation (NKE Schrödinger equation) for $E>V(E<V)$.

These equations uniformly have first time derivative. The forms of the equations embody the symmetry with respect to PKE and NKE. In this way, the NKE and PKE branches are treated on an equal footing. The modified equations remedy the logical inconsistency of the unmodified equations.

The reflection coefficient of a nonrelativistic particle encountering a onedimensional step potential with either infinite or finite width is calculated by means of Schrödinger equation and NKE Schrödinger equation. The results are physically reasonable, and have similar behavior with those of a relativistic particle.

Funding: This work was supported by the National Key Research and Development Program of China (2018YFB0704304-3).

Conflicts of Interest: The author declares no conflict of interest.

\section{References}

1. Schrödinger, E. Quantdsierung als Eiyenwertprobtem Ann. Phys. 1926, 384(4), 361 376; Schrödinger E. Quantisation as a Problem of Proper Values (Part I) in Collected Papers on Wave Mechanics; Blackie \& son Limited: London and Glasgow, 1928 1-12. 
2. Schrödinger, E. Quantdsierung als Eiyenwertprobtem Ann. Phys. 1926, 384(6), 489527; Schrödinger, E. Quantisation as a Problem of Proper Values (Part II) in Collected Papers on Wave Mechanics; Blackie \& son Limited: London and Glasgow, 1928 1340.

3. Schrödinger, E. Quantdsierung als Eiyenwertprobtem Ann. Phys. 1926, 385(13), 437490; Schrödinger, E. Quantisation as a Problem of Proper Values (Part III) in Collected Papers on Wave Mechanics; Blackie \& son Limited: London and Glasgow, 192863 101.

4. Schrödinger, E. Quantdsierung als Eiyenwertprobtem. Ann. Phy. 1926, 386(18), 109139; Schrödinger, E. Quantisation as a Problem of Proper Values (Part IV) in Collected Papers on Wave Mechanics; Blackie \& son Limited: London and Glasow, 1928 102123.

5. Klein, O. Quantentheorie und fünfdimensionale Relativitätstheorie. Zeitschrift für Physik Bd XXXVIL 1926, 59, 895-906.

6. Gordon, W. Der Comptoneffekt nach der Schrödingerschen Theorie. Zeitschrift für Physik Bd XL 1926, 9, 117-133.

7. Dirac, P. A. M. Relativity Quantum Mechanics with an Application to Compton Scattering. Proceedings of the Royal Society of London Series A 1926, 111, 405-424.

8. Schiff, L. I. Quantum Mechanics; $3^{\text {rd }}$ ed McGraw Hill Book Company: New York, 1968.

9. Berestetskii, V. B.; Lifshitz, E. M.; Pitaevskii, L. P. Quantum Electrodynamics Vol. 4 of course of Theoretical Physics; Pergmon Press: Bew York, 1982.

10. Wang, Huai-Yu New results by low momentum approximation from relativistic quantum mechanics equations and suggestion of experiments. J. Phys. Commun. 2020, 4, 12500 .

11. Klein, O. Die. Reflexion von Elektronen an einem Potentialsprung nach der relativistischen Dynamik von Dirac. Z. Physik 1929:, 53, 157-165.

12. Fuda, M. G.; Furlani, E. Zitterbewegung and the Klein paradox for spin-zero particles. Am. J. Phys. 1982, 50, 545-549.

13. Greiner, W.; Mueller, B.; Rafelski, J. Chapter 5 in Quantum Electrodynamics of Strong Field; Springer-Verlag: Berlin, 1985 112-121.

14. Yndurain, F. J. Relativistic Quantum Mechanics and Introduction to Field Theory; Springer-Verlag: Berlin, Heidelberg, 199687.

15. Holstein, B. R. Klein's paradox. Am. J. Phys. 1998, 66, 507-512.

16. Calogeracos, A.; Dombey, M. History and physics of the Klein paradox. Contem.

Phys. 1999, 40, 313-321.

17. Dombey, M.; Calogeracos, A. Seventy years of the Klein paradox. Phys. Rep. 1999, $315,41$.

18. Greiner W. Chapter 13 in Relativistic Quantum Mechanics-wave equations; 3rd ed. Springer-Verlag: Berlin, 2000325.

19. Mahan, G. D. Quantum Mechanics in a nutshell; Princeton University Press: Princeton, 2005356.

20. Gerritsma, R,; Kirchmair, G.; Zahringer, F. el al. Quantum simulation of the Dirac equation. Nature 2010, 463(08688), 68-71. 
21. Gerritsma, R.; Lanyon, B. P.; Kirchmair, G. et al. Quantum simulation of the Klein paradox with trapped ions. Phys. Rev. Lett. 2011, 106, 060503.

22. Wachter, A. Relativistic Quantum Mechanics; Springer Science+Business Media B. V. 2011 42-43.

23. Umul, Y. Z. A survey on Klein paradox. Optik 2019, 181, 258-263.

24. In [14] 25-27

25. Baym, G. Lectures on Quantum Mechanics; W. A. Benjamin, Inc.: New York, 1973 501.

26. Lelxe, F. L. Synthesis of Covariant Particle Equations. Phys. Rev. 1956, 2(2), 568581.

27. Paiano, G. Linear Potential with Relativistic Kinematics: Asymptotic Behaviour of the Eigenfunctions. IL NUOVO CIMENTO 1983, 70(4), 339-354.

28. Cea, P.; Colangelo, P.; Nardulli, G.; Paiano, G.; Preparata, G. WKB approach to the Schrödinger equation with relativistic kinematics. Phys. Rev. D 1982, 26(5), 1157-1167. 29. Cea, P.; Nardulli, G.; Paiano, G. Semiclassical treatment of the Schrodinger equation with relativistic kinematics and singular potential. Phys. Rev. D 1983, 28(9), 2291-2296.

30. Nickisch, I. J.; Durand, L.; Durand, B. Salpeter equation in position space: Numerical solution for arbitrary confining potentials. Phys. Rev. D 1984, 30(3), 660670.

31. Rosenstein, B.; Usher, M. Explicit illustration of causality violation: Noncausal relativistic wave-packet evolution. Phys. Rev. D 1987, 36(8), 2381-2384.

32. Lucha, W.; Schöber, F. F. Variational approach to the spinless relativistic Coulomb problem. Phy. Rev. D 1994, 50(8), 5443-5445.

33. Braua, F. Integral equation formulation of the spinless Salpeter equation. J. Math. Phys. 1998, 39(4), 2254-2263.

34. Hall, R. L.; Lucha, W.; Schöber, F. F. Energy bounds for the spinless Salpeter equation. J. Math. Phys. 2001, 42(11), 5228-5237.

35. Hall, R. L.; Lucha, W.; Schöber, F. F. Energy bounds for the spinless Salpeter equation: harmonic oscillator. J. Phys. A: Math. Gen. 2001, 34, 5059-5063

36. Hall, R. L.; Lucha, W.; Schöber, F. F. Convexity and potential sums for Salpetertype Hamiltonians. J. Math. Phys. 2002, 43(12), 5913-5925.

37. Brau, G. Upper limit on the number of bound states of the spinless Salpeter equation. Phys. Lett. A 2003, 313(4), 363-372.

38. Hall, R. L.; Lucha, W.; Schöber, F. F. Schrödinger upper bounds to semirelativistic eigenvalues. J. Phys. A: Math. Gen. 2005, 38, 7997-8002.

39. Li, Z. F.; Liu, I. J.; Lucha, W. et al. Relativistic harmonic oscillator. J. Math. Phys. 2005, 46, 103514.

40. Kowalski, K.; Rembielinski, J. Relativistic massless harmonic oscillator. Phys. Rev. A 2010, 81, 012118.

41. Kowalski, K.; Rembielinski, J. Salpeter equation and probability current in the relativistic Hamiltonian quantum mechanics. Phys. Rev. A 2011, 84, 012108.

42. Wang, Huai-Yu Solving Klein's paradox. J. Phys. Commun. 2020, 4, 125010.

43. Kowalski, K.; Rembieliński, J. The Wigner function in the relativistic quantum 
mechanics. Ann. Phys. 2016, 375, 1.

44. Wang, Huai-Yu Fundamental formalism of statistical mechanics and thermodynamics of negative kinetic energy systems. J. Phys. Commun. 2021, 5, 055012 .

45. Wang, Huai-Yu Macromechanics and two-body problems. J. Phys. Commun. 2021, 5,055018 . 